\title{
Artículos
}

\section{Adaptación de las editoriales españolas al libro electrónico}

\author{
Por Joana Costa-Knufinke
}

Resumen: El libro electrónico, entendido como un fichero digital, ha irrumpido en el panorama editorial español y ha provocado una gran variedad de cambios, tanto en el seno de las propias editoriales, como en la relación de las mismas con el resto de agentes de la cadena de valor tradicional del libro. Se describen estas transformaciones, además de los retos que tendrán que afrontar los editores frente a las nuevas exigencias de los e-books. Asimismo se propone una escala en cinco niveles para sistematizar el nivel de adaptación entre editoriales y libro electrónico. Su descripción detallada proporciona una panorámica actual de la situación de las editoriales españolas frente al e-book.

Palabras clave: Libro electrónico, Libro digital, E-book, Industria editorial, Digitalización, Cadena tradicional del libro, Edición electrónica, Edición digital.

\section{Title: Adaptation of the Spanish publishers to electronic book publishing}

Abstract: The electronic book, understood as a digital file, has entered the Spanish publishing scene and caused a variety of changes, both within publishing companies and in the relationship between the publishers and other players in the traditional book value chain. We describe these changes, in addition to the challenges that the publishers will face to meet the new e-books demand. In addition, to systematize the level of alignment between publishers and electronic books, a five-level scale is proposed. Its detailed description provides an overview of the current situation of Spanish publishers in relation to e-books.

Keywords: Electronic book, Digital book, E-book, Publishing, Digitization, Traditional book chain, Electronic publishing, Digital edition.

Costa-Knufinke, Joana. “Adaptación de las editoriales españolas al libro electrónico”. El profesional de la información, 2010, enero-febrero, v. 19, n. 1, pp. 13-20.

DOI: $10.3145 /$ epi.2010.ene.02

\section{Introducción}

Una de las novedades más importantes de la industria editorial en lo que va de siglo ha sido la aparición y popularización del libro electrónico o e-book. Éste ha sido el catalizador de importantes cambios que se estaban gestando en el sector editorial, como el surgimiento y la popularización de las plataformas de autopublicación, la posibilidad de realizar tiradas cortas con la "impresión bajo demanda", la lectura en soportes digitales como la pantalla del ordenador, el móvil, o los e-readers, o el nacimiento de editoriales exclusivamente electrónicas.

Todos estos cambios están produciendo transformaciones en la cadena de valor tradicional del libro que afectan a autor, lector, editor, distribuidor, librero y bibliotecario. Por este motivo se hace imprescindible un proceso de adaptación de todos sus componentes a la nueva situación.

Este artículo tiene un doble propósito. En primer lugar, analizar la influencia que la aparición del libro electrónico tiene sobre uno de los eslabones de la cadena tradicional del libro: las editoriales ${ }^{1}$. Y en segundo lugar, definir una escala de adaptación entre el libro electrónico y las editoriales españolas mediante la valoración de los factores clave en el proceso de implantación del e-book. La escala descrita aspira a realizar una radiografía del panorama actual y a sistematizar los estadios que concurren en el paso del libro analógico 
al digital en el seno de la industria editorial. A su vez pretende constituir una herramienta de ayuda para editoriales, librerías y bibliotecas, y crear una base sólida para futuras investigaciones.

\section{"El mundo editorial español está siguiendo un proceso poco uniforme en la implantación del libro electrónico"}

Cabe destacar que en España la edición en soportes distintos al papel ha aumentado de forma muy notoria durante los últimos años, y todo indica que continuará haciéndolo a un ritmo aún más vertiginoso² ${ }^{2}$.

\section{Aproximación al libro electrónico}

El término "libro electrónico" en lengua castellana ha sido víctima de una duplicidad de sentidos, pues se ha usado tanto para referirse al contenido, el archivo digital apto para la lectura en pantalla, como al continente, el soporte para la lectura. Medios de comunicación, expertos y profesionales del sector tienden a denominar a esta última acepción portalibros, lector digital, e-reader o dispositivo de lectura, y reservar el término libro electrónico o e-book para el archivo digital.

Este se puede presentar en más de veinte formatos distintos que están asociados en la mayoría de los casos a un dispositivo de lectura concreto. De esta diversidad de formatos destacamos el pdf de Adobe, un formato estático, y el EPUB también de Adobe, que es dinámico y ajustable al tamaño de la pantalla. Los libros electrónicos pueden presentar DRM (digital rights management), una tecnología de control de acceso que limita el uso de los e-books por parte de medios o dispositivos digitales con el fin de evitar la piratería.

Los libros electrónicos se pueden leer en una multiplicidad de dispositivos. Los tres más usados en la actualidad son la pantalla del ordenador personal, la pantalla del móvil y el e-reader dotado de tecnología de tinta electrónica.

Es importante destacar que en el contexto en que nos encontramos de estructuras de mercado globalizadas, los acontecimientos que tienen lugar en el mercado anglosajón, pionero en la implantación de nuevas tecnologías en la edición, influyen directamente en el español. El 8 de octubre de 2009 la prensa anunciaba la inminente puesta a la venta del e-reader de Amazon, el denominado Kindle Internacional, en cien países fuera de las fronteras de Estados Unidos, entre ellos España. Paralelamente la plataforma Google Books ha empe- zado a encontrar un hueco indiscutible en el panorama editorial español.

Partiendo de la experiencia anglosajona, muchos expertos y editores españoles coinciden en opinar que la implantación generalizada del e-book surgirá tras la necesaria popularización de los dispositivos de lectura o $e$-readers ${ }^{3}$.

\section{"La Red alberga multitud de proyectos menores nacidos con espíritu digital"}

\section{3. Época de cambios en España}

El Gobierno español ha procurado apoyar a las editoriales en el proceso de cambio y adaptación del libro físico al libro electrónico a fin de dotarlas de estrategias útiles y de acortar las diferencias con los países anglosajones. Entre los medios aportados cabe mencionar: las ayudas económicas del Ministerio de Cultura a las editoriales para promocionar la digitalización de libros; las investigaciones realizadas para facilitar la adaptación de las editoriales al libro electrónico; y la subvención de proyectos específicos como el programa Enclave. Este proyecto reúne a la Federación de Gremios de Editores de España y a la Biblioteca Nacional y tiene el propósito de impulsar la digitalización y la puesta a la venta de fondos sujetos a derechos de autor.

\section{Cambios en el seno de la editorial}

Para adaptarse con eficacia al libro electrónico, las editoriales deberán digitalizar en un formato óptimo para e-readers, móviles y pantallas de ordenador todo o parte de su catálogo editorial, haciéndolo accesible para el lector desde bibliotecas y librerías. Asimismo deberán crear archivos digitales óptimos de sus novedades editoriales, para lo que el Ministerio recomienda el uso del formato EPUB. Esta es una operación con un coste medio aproximado de 100 euros por volumen.

La mayoría de contratos antiguos de la época papel no consideraban la cesión de derechos digitales, pero los nuevos formatos, soportes y canales, y las nuevas formas de explotación exigen una regulación distinta de los derechos de autor que debe ser incluida en los contratos de cesión de derechos de la editorial. Por este motivo los editores también se verán obligados a adaptar los derechos de autor de los contratos antiguos mediante la inclusión de una cláusula o la firma de una adenda que regule las nuevas formas de explotación digitales. 
Con la venta del libro electrónico los costes generales de producción para el editor se abaratan porque se eliminan los gastos de impresión y almacenaje y se reducen los de distribución. En consecuencia se puede disminuir considerablemente el precio de venta al público del producto final. Para que el autor pueda recibir la misma percepción por libro vendido en el formato tradicional y en el digital, el porcentaje de derechos que debe percibir es mayor que el de la venta en papel. Por este motivo los derechos de autor digital se encuentran la mayoría de la veces sobre el $25 \%$, en contra de la media del $8 \%$ del formato papel.

Otro de los retos que deberán afrontar los editores es la lucha contra la piratería porque este fenómeno imparable ya está afectando a la industria incluso antes de que ésta se haya adaptado totalmente a los nuevos formatos. Una de las estrategias posibles es el uso del citado DRM, que hace la piratería algo menos alcanzable. Otra técnica eficaz puede ser la creación de libros electrónicos enriquecidos; es decir, libros que aprovechen las posibilidades hipertextuales y los instrumentos multimedia como audio y vídeo, para dar un valor añadido respecto al libro en papel y crear un producto más competitivo y más difícil de piratear.

\section{"En 2004 Google anunció que pretendía escanear los treinta y dos millones de libros existentes, $y$ dar acceso libre a todos ellos desde Google Books"}

Para llevar a cabo todos estos pasos será imprescindible preparar al equipo editorial para su adaptación a los nuevos requerimientos de la era digital: deben disponer de los medios para interactuar con el resto de agentes de la cadena del libro como libreros o bibliotecarios que, ante la nueva panorámica, han generado y generarán nuevas expectativas.

Del mismo modo, afrontar los gastos generados por los cambios mencionados supondrá una elevada inversión económica. Las empresas deberán definir un modelo de negocio con el que recuperar la inversión realizada. Para ello será oportuno dirigirse a nuevos públicos y aprovechar las posibilidades del mercado ofrecidas por el elevado y creciente número de internautas que dominan la lengua castellana, una lengua con más de 450 millones de hablantes en todo el mundo. Puede resultar favorable para las editoriales experimentar con nuevos modelos comerciales como la venta por capítulos, el préstamo digital a bibliotecas o directamente a lectores, y sacar el máximo provecho posible de las nuevas formas de promoción ofrecidas por los libros electrónicos, como por ejemplo permitir al usuario hojear los contenidos en línea o buscar en ellos palabras $\mathrm{y}$ frases concretas.

\section{Cambios en relación con el resto de integrantes de la cadena}

La irrupción del libro electrónico en el panorama editorial ha provocado un cambio de paradigma que exigirá la adaptación de todos los integrantes de la cadena de valor tradicional del libro.

\section{"El editor pierde su rol como único proveedor de contenidos y la posición de liderazgo en la cadena por el auge de las plataformas de edición"}

La posición del editor en relación a los demás integrantes es contradictoria. Por una parte pierde su rol como único proveedor de contenidos y pierde la posición de liderazgo en la cadena porque el auge de las plataformas de autoedición como Bubok o Lulu pone la edición de contenidos al alcance de todos.

En este sentido se produce una "democratización" de la edición en la que los roles de autor y editor, separados claramente en la cadena tradicional del libro, se convierten en un solo agente. Contrariamente, el papel tradicional de "filtro" del editor es más necesario que nunca ante el aumento exponencial de la oferta de contenidos, pues la calidad del producto final de los libros autoeditados sólo está garantizada por el propio autor.

\section{http://www.bubok.com http://www.lulu.com}

Paralelamente la entrada del libro electrónico provoca una pérdida de peso específico del impresor y el distribuidor, dos agentes que dejan de ser necesarios con el uso de las nuevas técnicas de distribución digital. Asimismo, tal y como está pasando en los países anglosajones y en otros países europeos, la nueva situación facilita la entrada de nuevos actores en la cadena, como grandes compañías de telefonía o los denominados agregadores de contenidos, agentes que proporcionan un servicio de acceso a múltiples libros electrónicos publicados por diferentes editores desde una misma plataforma.

El librero y el bibliotecario podrán aprovechar esta nueva situación para realizar tratos directamente con las editoriales o los agregadores de contenidos que puedan, en última instancia, beneficiar al lector. 
Ante esta nueva situación el editor deberá valorar el beneficio de proteger el rol tradicional de todos los componentes de la cadena, como el distribuidor y el librero, o bien abrirse a nuevas formas de comercio como la venta de contenidos desde su propia plataforma online.

\section{Criterios de sistematización del nivel de adaptación}

Hemos considerado importantes los siguientes parámetros para determinar el nivel de adaptación de las editoriales al libro electrónico:

- porcentaje de contratos de derechos de autor de la editorial adaptados al formato digital, tanto del fondo como de las obras de nueva contratación;

- porcentaje de libros digitalizados del fondo editorial, y el número de formatos en que se han digitalizado;

- porcentaje de libros a la venta en la Red en formato digital;

- número de plataformas de venta por cada $e$-book, por ejemplo desde sitios web de bibliotecas y/o de librerías;

\section{"Los nuevos formatos, soportes, canales y formas de explotación exigen una regulación distinta de los derechos de autor"}

- número de plataformas de visualización por cada libro electrónico, por ejemplo mediante sitios web de bibliotecas y/o de librerías;

- número de e-readers con que son compatibles los libros digitalizados disponibles;

- porcentaje de libros que efectúan una salida simultánea en papel y en formato electrónico;

- porcentaje de la fuerza de trabajo total de la editorial dedicada al libro digital;
- porcentaje de la facturación del canal digital en comparación con el total de la facturación;

- porcentaje de libros digitalizados que presentan "contenido enriquecido".

\section{Niveles de adaptación}

A partir de los parámetros descritos hemos realizado la escala de adaptación en cinco niveles.

\section{Nivel 1. Editoriales en stand by}

En este nivel encontramos las empresas que aparentemente no han realizado ningún tipo de cambio en sus estrategias para adaptarse al libro electrónico. A modo de ejemplo, a 20 de diciembre de 2009, los sitios web de las siguientes editoriales no hacen mención alguna a la venta de libros electrónicos: Acantilado, La otra orilla, Melusina, Libros del Asteroide e Impedimenta. Asimismo ninguno de sus libros se puede visualizar ni comprar en formato electrónico en la Red.

\section{Nivel 2. Editoriales en vías de adaptación}

Reúnen la característica común de haber realizado algunos pasos en el seno de la empresa para adaptarse al libro electrónico, aunque estos aún no han tenido efecto de cara al lector o consumidor final. En este nivel encontramos la plataforma formada por los grupos Planeta, Santillana y Random House Mondadori (ver cuadro 1). Según la información aparecida en la prensa, podemos deducir que la intención de esta plataforma es actuar de agregador de los contenidos de otras editoriales de la industria española, hecho que facilitará el acceso a grandes paquetes de contenido por parte de bibliotecarios, libreros y, en último término, lectores.

\section{Nivel 3. Editoriales parcialmente adaptadas}

No han empezado a digitalizar su catálogo editorial por su cuenta, pero han hecho acuerdos con terceros para que digitalicen sus libros y permitan su compra y/o visualización.

\section{- Tratos con Publidisa}

Publidisa es una empresa de comunicaciones que nació el año 2000 con la intención de dar servicios de print on demand y de venta de libros electrónicos a editores españoles y sudamericanos. Para ello creó una

\begin{tabular}{|l|c|c|c|c|c|c|c|}
\hline Plataforma & Formato & Política de precio & $\begin{array}{c}\text { Lugar de } \\
\text { venta }\end{array}$ & $\begin{array}{c}\text { Fecha prevista } \\
\text { de entrada en } \\
\text { funcionamiento }\end{array}$ & $\begin{array}{c}\text { ¿Distribución } \\
\text { exclusiva de } \\
\text { e-books? }\end{array}$ & $\begin{array}{c}\text { ¿Abierta } \\
\text { a más } \\
\text { editoriales? }\end{array}$ & $\begin{array}{c}\text { Posibles } \\
\text { socios } \\
\text { futuros }\end{array}$ \\
\hline $\begin{array}{l}\text { Planeta, } \\
\text { Santillana } \\
\text { y Random } \\
\text { House } \\
\text { Mondadori }\end{array}$ & EPUB & $\begin{array}{c}\text { Inferior al } 70 \% \\
\text { del pvp del libro } \\
\text { impreso }\end{array}$ & $\begin{array}{c}\text { Sitios web } \\
\text { de librerías }\end{array}$ & $\begin{array}{c}\text { Abril de } 2010 \\
\text { (Feria del libro } \\
\text { de Madrid) }\end{array}$ & Sí & Sí & $\begin{array}{c}\text { Grupo } \\
\text { Anaya }\end{array}$ \\
\hline
\end{tabular}

Cuadro 1. Características de la principal plataforma que contiene editoriales en vías de adaptación (nivel 2) 
plataforma online de venta de libros y revistas electrónicos denominada Todoe-book. Por el precio aproximado de $10 €$ por volumen, Publidisa escanea en formato pdf los libros cedidos por la editorial y les aplica el DRM de Adobe. Posteriormente los comercializa directamente en Todoe-book y los distribuye mediante la red de Librerías Asociadas a Publidisa (LAP), entre las que encontramos El Corte Inglés, La Casa del Libro, o la librería Gandhi. Tiene a la venta más de mil títulos de unas trescientas editoriales de libros y revistas como Edaf, Antoni Bosch SA o L'Avenç.

http://www.publidisa.com

http://www.todoe-book.com

http://www.elcorteingles.es

http://www.casadellibro.com

http://www.gandhi.com.mx

“El rol tradicional de 'filtro' del editor es más necesario que nunca ante el aumento exponencial de la oferta de contenidos"

\section{- Tratos con Google}

En 2004 Google anunció que pretendía escanear los treinta y dos millones de libros que existen en el planeta, crear libros electrónicos de todos ellos y darles acceso libre desde su web Google Books (Google Libros en la versión castellana). Cinco años más tarde las cifras oficiales indican que ha escaneado más de siete millones de obras. Para escanear las que tienen derechos de autor vigentes, Google realiza tratos con las editoriales: les ofrece la digitalización gratuita a cambio de un ejemplar físico o digital de cada uno de los libros que desee hacer parcialmente visible en la web (normalmente se puede visualizar el 20\% del documento). Un mínimo de 85 editoriales españolas, entre las que dominan las científico-técnicas, han cedido parte o la totalidad de su fondo editorial para que sea parcialmente visualizable en la web de Google. Entre ellos destacamos Rialp y Paidotribo.

http://www.books.google.com

Google activará próximamente el nuevo proyecto Google Editions, un sistema de visualización completa online de libros sujetos a derechos de autor mediante distintas modalidades de pago.

\section{- Tratos con la Biblioteca Nacional}

En la Feria del Libro de Madrid de octubre de 2009 se anunció que la Biblioteca Nacional digitalizará 1.350 obras sujetas a derechos de autor de un total de 90 editoriales pequeñas, medianas y grandes, agrupadas bajo el Proyecto Enclave. A razón de unas veinte obras por editor, todas ellas se pueden consultar, ojear y/o comprar en la web de la Biblioteca Nacional desde

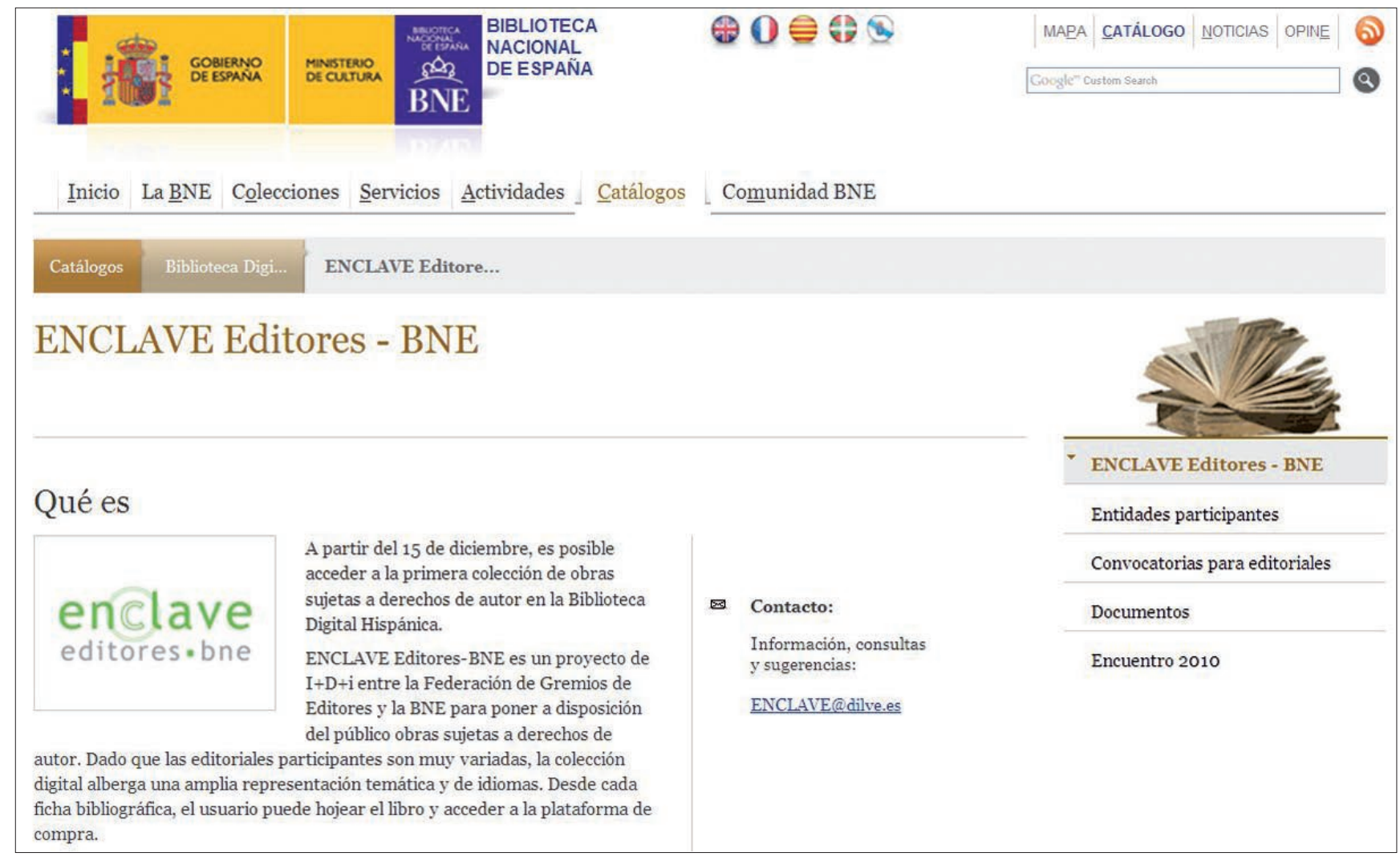

http://www.bne.es/es/Catalogos/BibliotecaDigital/enclave/ 
el día 15 de diciembre de 2009 y con condiciones establecidas por cada uno de los editores. De este modo, la Biblioteca Nacional aumentará su oferta de libros electrónicos a obras sujetas a derechos de autor.

- Tratos con agregadores de contenidos

Un número creciente de editoriales españolas está comercializando parte de los libros de su catálogo editorial mediante plataformas de venta formadas por otras editoriales. Éstas, a su vez, aglutinan los contenidos del máximo número de editoriales posible. Por ejemplo una selección del catálogo de Maeva Ediciones se puede comprar desde la plataforma Leqtor (cuadro 2).

\section{Nivel 4. Editoriales adaptadas al libro electrónico}

En este nivel situamos las editoriales tradicionales que han dado un paso al frente para adaptarse plenamente al $e$-book. En primer lugar encontramos las editoriales jurídicas y del sector de la lingüística, las pioneras del mercado español (e internacional) en empezar a realizar su venta de contenidos en formato electrónico. Entre ellas destacamos La Ley y Aranzadi. Cabe destacar también la experiencia pionera de Edi.cat, que nació en abril de 2009 con la intención de convertirse en el gran portal de venta de libros electrónicos en catalán mediante la incorporación de $e$-books de otras

\begin{tabular}{|c|c|c|c|c|c|}
\hline $\begin{array}{l}\text { Plataforma } \\
\text { (editor) }\end{array}$ & Formato(s) & $\begin{array}{l}\text { Política de } \\
\text { precios }\end{array}$ & $\begin{array}{c}\text { E-books a la } \\
\text { venta en: }\end{array}$ & $\begin{array}{l}\text { Fecha de } \\
\text { comienzo }\end{array}$ & $\begin{array}{c}\text { Libros editados } \\
\text { (todos en blanco y } \\
\text { negro) }\end{array}$ \\
\hline $\begin{array}{l}\text { Edi.cat } \\
\text { (Cossetània } \\
\text { Edicions, } \\
\text { Edicions } \\
\text { Bromera y } \\
\text { Angle Editorial) }\end{array}$ & $\begin{array}{l}\text { PRC de } \\
\text { Mobipocket } \\
\text { con DRM de } \\
\text { Mobipocket y } \\
\text { EPUB y pdf con } \\
\text { DRM de Adobe } \\
\text { Digital Editions. }\end{array}$ & $\begin{array}{l}3 \text { gamas de } \\
\text { precios. } \\
\text { Novedades: } \\
60 \% \text { de } \\
\text { descuento } \\
\text { respecto al } \\
\text { homólogo en } \\
\text { papel } \\
\text { Backlist: } 4,95 € \\
\text { Obras libres de } \\
\text { derechos: } 1 €\end{array}$ & $\begin{array}{l}\text { http://www. } \\
\text { edi.cat } \\
\text { o bien pago } \\
\text { en librerías } \\
\text { y descarga } \\
\text { mediante } \\
\text { código en la } \\
\text { misma web }\end{array}$ & $\begin{array}{c}\text { Abril } \\
\text { de } 2009\end{array}$ & $\begin{array}{l}\text { Los libros de las tres } \\
\text { editoriales fundadoras y } \\
\text { de La Galera y Tandem, } \\
\text { entre otras. }\end{array}$ \\
\hline $\begin{array}{l}\text { 36-L Books } \\
\text { (Abacus, } \\
\text { Vicenç Vives, } \\
\text { Ferran Soriano } \\
\text {-Presidente } \\
\text { de Spanair-, } \\
\text { y Ernest Folch } \\
\text {-director de } \\
\text { Grup Cultura } \\
\text { o3) }\end{array}$ & $\begin{array}{l}\text { EPUB con DRM } \\
\text { de Adobe Digital } \\
\text { Editions. }\end{array}$ & $\begin{array}{l}\text { Descuento } \\
\text { variable entre } \\
\text { el } 15 \text { y el } 50 \% \\
\text { respecto a su } \\
\text { homólogo en } \\
\text { papel. }\end{array}$ & $\begin{array}{l}\text { LEQTOR } \\
\text { http://www. } \\
\text { leqtor.com }\end{array}$ & $\begin{array}{l}3 \text { de } \\
\text { diciembre } \\
2009\end{array}$ & $\begin{array}{l}\text { Aglutinan libros de las } \\
\text { siguientes editoriales: } \\
\text { Ara Llibres } \\
\text { Grup } 62 \\
\text { Planeta } \\
\text { RBA } \\
\text { Maeva Ediciones } \\
\text { Roca Editorial } \\
\text { Ediciones Luciérnaga } \\
\text { El Aleph Editores } \\
\text { Etc. }\end{array}$ \\
\hline $\begin{array}{l}\text { Ambook } \\
\text { (Urano) }\end{array}$ & $\begin{array}{l}\text { EPUB con DRM } \\
\text { de Adobe Digital } \\
\text { Editions. }\end{array}$ & $\begin{array}{l}\text { Descuento } \\
\text { aprox. del } 25 \% \\
\text { respecto a su } \\
\text { homólogo en } \\
\text { papel. }\end{array}$ & $\begin{array}{l}\text { http://www. } \\
\text { amabook.com }\end{array}$ & $\begin{array}{c}10 \mathrm{de} \\
\text { diciembre } \\
2009\end{array}$ & $\begin{array}{l}\text { Novedades y backlist } \\
\text { de la editorial } \\
\text { Urano y previsión } \\
\text { de incorporar libros } \\
\text { de otras editoriales } \\
\text { independientes. }\end{array}$ \\
\hline Leer-E & $\begin{array}{l}\text { PRC de } \\
\text { Mobipocket, EPUB } \\
\text { y/o pdf, según el } \\
\text { título. }\end{array}$ & $\begin{array}{l}\text { Predomina } \\
\text { la gama de } \\
\text { precios entre } 2 \\
\text { y } 5 € .\end{array}$ & $\begin{array}{l}\text { http://tienda. } \\
\text { leer-e.es }\end{array}$ & 2006 & $\begin{array}{l}\text { Traducciones propias de } \\
\text { libros libres de derechos } \\
\text { de autor y libros de } \\
\text { más de } 60 \text { editoriales. } \\
\text { 'Colección Palabras } \\
\text { mayores' mediante } \\
\text { pacto con la Agencia } \\
\text { Literaria Carmen } \\
\text { Balcells. Incluye títulos } \\
\text { de Julio Cortázar, Juan } \\
\text { Marsé y Mario Vargas } \\
\text { Llosa, entre otros. }\end{array}$ \\
\hline Luarna & $\begin{array}{l}\text { Pdf de Adobe sin } \\
\text { DRM para pantalla } \\
\text { de } 6 \text { pulgadas y } \\
\text { para pantalla de } 8 \\
\text { a } 10 \text { pulgadas. }\end{array}$ & $\begin{array}{l}\text { Predomina } \\
\text { la gama de } \\
\text { precios entre } \\
2,80 € \text { y } 3,80 € .\end{array}$ & $\begin{array}{l}\text { http://www. } \\
\text { luarna.com }\end{array}$ & - & $\begin{array}{l}\text { Ficción y no ficción, } \\
\text { con especial énfasis } \\
\text { en manuales sobre las } \\
\text { nuevas tecnologías. }\end{array}$ \\
\hline
\end{tabular}

Cuadro 2. Características de las principales editoriales adaptadas al libro electrónico (nivel 4) y de las editoriales electrónicas (nivel 5) 
editoriales (cuadro 2). El objetivo de esta plataforma, y de otras que se encuentran en este mismo nivel como Leqtor o Amabook, es el de convertirse en agregadores exclusivos o no exclusivos de los contenidos de otras editoriales para servir sus productos a librerías, bibliotecas y/o lectores (cuadro 2).

http://www.laley.es/content/Inicio.aspx

http://www.aranzadi.es

http://www.edi.cat

\section{Nivel 5. Editoriales electrónicas}

En este nivel situamos las editoriales nacidas con el espíritu de ser únicamente electrónicas. Por este motivo su nivel de adaptación al libro electrónico es de entrada máximo. Entre las plataformas más destacables encontramos Leer-E y Luarna (cuadro 2). Del mismo modo, el servicio de autopublicación y plataforma de impresión bajo demanda Bubok se ha convertido en editorial electrónica mediante la creación de la biblioteca $B A T$ (Biblioteca Andreu Teixidor), un sello avalado por el propio editor y accionista de la empresa, y dedicado a rescatar libros descatalogados.

\section{http://www.bubok.com}

En la Red se albergan multitud de proyectos menores nacidos también con espíritu digital como Badosa, Noveles.com, Proscritos la Editorial, Blognovelas.es, Soopbook o Bookandyou.

http://www.badosa.com

http://www.noveles.com

http://www.proscritoseditorial.com

http://www.blognovelas.es

http://www.soopbook.com

http://www.bookandyou.com

\section{Un proceso irregular}

El sector editorial español está siguiendo un proceso poco uniforme en la implantación del libro electrónico. Por ejemplo, no hay uniformidad de criterios sobre cómo se va a recuperar el coste invertido en formación del personal, digitalización del fondo, creación de nuevos sitios web, lucha contra la piratería y redefinición de los ámbitos comerciales, entre otros gastos. Cada plataforma o editorial que ha dado algún paso hacia el proceso de adaptación o que ha nacido con espíritu electrónico está usando sus propios criterios de precios, de formatos a disposición del lector, de uso o no de DRM, o su propia política de tratos con libreros y bibliotecarios.

En todos los niveles de adaptación descritos, el libro electrónico se entiende generalmente como un mero paso del contenido del papel a la pantalla, y queda en un segundo término el uso de todas las posibilidades multimedia ofrecidas por las nuevas tecnologías. Los libros ilustrados están al margen de los proyectos de digitalización porque aún no son aptos para ser visualizados en los lectores digitales del mercado, todos aún en $b / n$.

Es muy probable que la comercialización de los dispositivos de lectura, cada vez más numerosos en nuestro país, sumada a la salida al mercado de los contenidos digitales de varias editoriales españolas en los próximos meses, dinamice y popularice el comercio del libro electrónico en España. Esto significará un paso más de la revolución digital que se aventura imparable.

\section{Consideraciones finales}

Ante el reto que implica la comercialización del libro electrónico, el presente artículo apunta como recomendable para las editoriales la valoración de las siguientes consideraciones:

- Estudio y aplicación comedida de las experiencias pioneras en el mercado internacional (con Estados Unidos a la cabeza) y en el mercado nacional (Planeta, Edi.cat, etc.), así como de la experiencia de la industria de la música.

- Sacar partido de las incuestionables ventajas que el e-book puede aportar al mercado editorial, como la apertura de un nuevo canal de distribución inmediato, permanente y de gran crecimiento, la actualización constante de los contenidos, y el cross publishing, es decir, la edición de un mismo contenido en diferentes formatos según las necesidades del mercado.

- Aprovechar las nuevas tecnologías para crear libros electrónicos que respondan a las expectativas de los nuevos lectores. La elaboración de productos complejos que usen todos los instrumentos multimedia disponibles puede ayudar a su vez a evitar la piratería.

- Calibrar el alcance de la oferta de empresas como Google Books o Amazon limitándola a una parte del catálogo y usándola como prueba de la demanda real del mercado a los libros ofrecidos por la editorial.

\section{Una mirada hacia el futuro}

El sector del libro y de las nuevas tecnologías es un ámbito dinámico sujeto a permanentes cambios. Es continua la salida al mercado de novedades en dispositivos, la aprobación de nuevas políticas y la apertura de mercados por parte de empresas anglosajonas y asiáticas, acontecimientos que abren nuevas vías, reflexiones y dudas en el marco teórico y práctico de este campo de estudio.

Este dinamismo dificulta la sistematización de la interacción entre libro electrónico y editoriales, a la vez que nos exige introducirnos en nuevos ámbitos de 
investigación académica de los que podrán surgir propuestas para mejorar el ajuste de las editoriales españolas al libro electrónico. En último término, esto ayudará a todos los agentes de la cadena tradicional del libro a culminar su proceso de adaptación a la nueva situación requerida por la era digital.

\section{Notas}

1. El presente escrito no ha incluido mención alguna a las editoriales especializadas en libro de texto porque estas responden a dinámicas subordinadas a factores que salen de los límites de nuestro propósito.

2. Según el trabajo El libro y las nuevas tecnologías. El libro electrónico realizado en 2009 por el Ministerio de Cultura, la edición electrónica en España "ha tenido un aumento espectacular en los últimos años, pasando del 3,23\% del total editado en el año 2000 (2.011 ISBNs) al 8,1\% en 2008 (8.447 nuevos títulos)"”.

3. Según el informe Digitalización del libro en España realizado por Dosdoce y Ediciona "[el] 80\% de los encuestados considera que la inminente comercialización en España de soportes de lectura de libros electrónicos como Sony e-Reader, Kindle o I-Liad impulsará la digitalización del libro en España".

\section{Bibliografía y recursos consultados}

Alós, Ernest. "La oferta de e-books en castellano y catalán se dispara en diciembre". El periódico, 11 de octubre de 2009.

http://www.elperiodico.com/default.asp? idpublicacio_PK=46\&idioma $=C$ AS\&idnoticia_PK=652035\&idseccio_PK=1013

Dosdoce.com y Ediciona. Digitalización del libro en España. 2008 http://www.dosdoce.com/continguts/estudios/contacto_cas.php?ID=22

EFE. "La Biblioteca Nacional digitalizará 1.350 obras sujetas a derechos de autor". El mundo, 12 de octubre de 2009.

http://www.elmundo.es/elmundo/2009/10/08/navegante/1255010900.html

García-Marco, Francisco-Javier. "El libro electrónico y digital en la ecología informacional: avances y retos". El profesional de la información, 2008, julio-agosto, v. 17, n. 4, pp. 373-389.

García-Velayos, Jorge. "Los derechos de autor, el complicado reparto..." Delibros, 2009, junio, 232, pp. 20-26.
Geli, Carles. "Frankfort se rinde al libro electrónico". El país, 14 de octubre de 2009.

http://www.elpais.com/articulo/cultura/Francfort/rinde/libro/electronico/ elpepicul/20091014elpepicul_1/Tes

Gil, Manuel; Jiménez, Francisco-Javier. El nuevo paradigma del sector del libro. Madrid: Trama Editorial, 2008.

Ministerio de Cultura. El libro y las nuevas tecnologías. El libro electrónico. 2007.

http://www.mcu.es/libro/docs/MC/CD/libro_tecnologias2007.pdf

Ministerio de Cultura. Panorámica de la edición española. 2008. http://www.mcu.es/libro/MC/PEE/index.html

Ministerio de Cultura. El libro y las nuevas tecnologías. El libro electrónico, 2009.

http://www.mcu.es/libro/docs/MC/CD/LIBROELECTRONICO.pdf

Ministerio de Industria, Turismo y Comercio. Informe de diagnóstico de los desafíos pendientes en el ámbito de la industria editorial española. 2008. http://www.proyectocaliope.es/informes/informe01.pdf

Martín-González, Juan-Carlos; Pivetta, Emma. "Factores clave en el proceso de adquisición de libros electrónicos". El profesional de la información, 2008, julio-agosto, v. 17, n. 4, pp. 408-413.

Millán, José-Antonio. "El polimorfo libro electrónico". El profesional de la información, 2008, julio-agosto, v. 17, n. 4, pp. 369-372.

Mora, Rosa; Geli, Carles. "Arranca la primera gran batalla por la distribución del libro digital". El país, 3 de julio de 2009.

http://www.elpais.com/articulo/cultura/Primera/gran/batalla/distribucion/ libro/digital/elpepucul/20090703elpepicul_2/Tes

Rodríguez, Joaquín. Edición 2.0. Los futuros del libro. Barcelona: Melusina, 2007.

Seminario "Innovación tecnológica y digitalización en el sector editorial". Gremio de Editores de Catalunya y Cedro. Barcelona, 14 de setiembre de 2009.

Thompson, John B. Books in the digital age. Cambridge, UK; Malden, EUA: Polity Press, 2005.

Joana Costa-Knufinke, Universitat de Barcelona joanacostak@gmail.com

\section{Próximos temas centrales}

Marzo 2010

Mayo 2010

Julio 2010

Septiembre 2010

Noviembre 2010

Enero 2011

Marzo 2011

Mayo 2011
Publicidad y comunicación empresarial

Información bio-médica

Arquitectura de la información

Cooperación de bibliotecas en red

Medios de comunicación en internet

Psicología y sociología de la información

Archivos administrativos e intranets

Información de las administraciones públicas

Los interesados pueden remitir notas, artículos, propuestas, publicidad, comentarios, etc.,

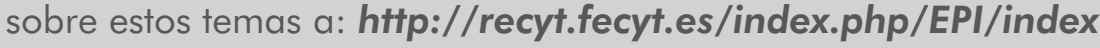

